\title{
The Persuasion Knowledge Model Within Instagram Advertisements
}

\author{
Natalia Miksa ${ }^{1}$ and Robert Hodgson" \\ ${ }^{1}$ McDowell High School, Erie, PA, USA \\ "Advisor
}

\begin{abstract}
The aim of this research was to find a correlation between the Persuasion Knowledge Model and Instagram Advertisements. Past research has primarily focused on this model on other forms of media such as television, radio, newspaper, etc. Additionally, the ages studied have been over the age of 30 as well as not focused within a certain geological demographic. For this study, participants were residents of Pennsylvania who vary in age between 18-25 years old. Using a survey, statistical analysis, and Pearson Product Moment Correlation Coefficient Analysis test (PCC test), this study measured each of the 49 participants' persuasion knowledge and correlated it to the advertisements chosen in the survey. The analyses found that those with lower persuasion knowledge were more likely to select an advertisement with less text variance and influential text. Further, a new understanding was found that showed a slightly negative correlation due to the PCC test, but the correlation was not statistically significant. Therefore, the findings of this research study refuted the initial hypothesis and other studies conducted in the field of persuasion knowledge.
\end{abstract}

\section{Introduction}

The online marketplace has become a globalized industry, especially within the past few decades (Abdelaal, 2014). Additionally, advertising appeals have been evident in the advertising arena (Wu, 2009). Advertising is mass media content intended to persuade audiences and viewers to take action on products, services, and ideas (Mohapatra et al., 2016). The primary goal of advertising is to drive consumer behavior in a particular way regarding a specific product, service, or concept (Abdelaal, 2014). Under the umbrella of advertising is what is known as the Persuasion Knowledge Model. This model referred to how a customer felt in regards to theories surrounding persuasion, including beliefs about marketers' motives, strategies, and tactics (Campbell et al., 2000). A previous research study discovered that persuasion knowledge leads to attitude formation and can subsequently influence behavior. (Mikolajczak-Degrauwe and Bregman, 2013). Hence, technological awareness, or being aware of persuasion knowledge techniques, among customers was expected to positively impact market growth (Grand View Research, 2020). The study results determined that further research should examine other possible mediators of this relationship (Mikolajczak-Degrauwe and Bregman, 2013).

The evolution of social media has been fueled by human nature and the desire to communicate and advance in digital technology (Maryville University, 2021). Social media has become the most prominent form of communication and advertising (Maryville University, 2021). More specifically, the media plays an important role involving persuasion knowledge by accessibly broadcasting advertisements to target audiences. Instagram, a free downloaded application that allows users to interact with photos and/or products, is a universal form of advertisement sharing (Kus, 2018). Instagram users commonly present persuasion techniques within their online advertisements.

This paper intends to explore the relationship between consumer product involvement and product knowledge and deepen the understanding of the correlation between Instagram advertising and the Persuasion Knowledge Model in young adults. 


\section{Literature Review}

\subsection{E-commerce and Instagram Advertising}

Electrical commerce, or e-commerce, can be defined by the commercial transactions that are electronically conducted through the use of the internet (Direct Packaging, 2020). As technology increases, so does the e-commerce market (Direct Packaging, 2020). There has been a 313\% increase in online shopping sales in the past decade, with approximately 517.4 billion dollars in 2018 alone (Direct Packaging, 2020). Its 24-hour convenience allows shoppers to purchase items wherever and whenever easily, which provides ideal conditions for online stores. The internet enables enterprises to provide more product information online at a cost far lower than other conventional forms of popular media, such as newspapers, magazines, and television (Direct Packaging, 2020). It also allows brands to manufacture, market, and sell products in addition to providing customer services in an efficient and persuasive manner (Wan-Yu Liu, 2012). In other words, the e-commerce market is continually growing and is likely to be the most profitable way of shopping in the coming decades (Columbia University Public Health, 2019).

In recent years, Instagram has been an essential part of the e-commerce community (Kus, 2018). Within internet advertising on social media, it is known that Instagram is an effective way to promote ideas and products to a variety of age groups (Kus, 2018). Stand-alone companies have the opportunity to showcase and advertise their brand via Instagram stories and sponsored advertisements (Kus 2018).

The primary age group that uses Instagram as their main source of social media is 18-25 years. Compared to Facebook whose user age range is from 27-55, Instagram is a social media platform tailored to the newer generations (Omnicore, 2019). In order to target their largest demographic, retailers on Instagram tend to heavily focus on young adults and teenagers (Omnicore, 2019). Overall, there is a need in this research field for studies to strictly discuss young adult audiences and the persuasion knowledge employed through Instagram advertisements.

\subsection{Advertisements In Social Media}

As mentioned earlier, advertisements have been extraordinarily helpful when promoting a business because they can then analyze the factors of the advertisement that work best with consumers (Wan-Yu Liu, 2012). A common approach, known as a tracking study, surveys members of the target audience over a specified period of time (Saleh, 2018). This approach allows researchers to track ongoing changes in their attitudes, purchase intent, and brand knowledge (Saleh, 2018). Companies aim to reduce costs, shorten product life cycles, and speed up customer feedback to improve service quality (Wan-Yu Liu, 2012). For instance, many online businesses promote free shipping with orders over a specified amount of money. Additionally, companies who advertise on social media often use personal data to keep up with trending and in-demand items the viewers are currently searching for (Wan-Yu Liu, 2012). Using strategic placement and designs along with user information, experts are able to make advertisements that will both appeal to the viewer and subconsciously prompt the viewer to remember the item. One of the main ways that an advertisement can gain traction is when the seller persuades the buyer to look at their products, primarily through the use of influential text (Wan-Yu Liu, 2012). Influential text is the verbiage and language used by marketers that have a persuasive connotation. Common tactics in developing the influential text are to create a limited supply to increase the demand for a product. Examples of text that utilize persuasive tactics include "flash sale" and "limited availability." Overall, the ultimate goal as a retailer is for the shoppers to interact with their advertisements in order to increase their profits.

\subsection{Persuasion Knowledge Model}


The Persuasion Knowledge Model (PKM), first introduced in 1994, is focused on a user's knowledge of persuasion motives and tactics to interpret, evaluate, and respond to influence attempts from marketers and others (Friestad and Wright, 1994). Further, the Persuasion Knowledge Model has impacted the way researchers assess advertisements by allowing them to look closer at the motives behind the model (Friestad and Wright, 1994). Resourceful participants with high persuasion knowledge have the ability to select response tactics from their repertoire, similar to the way agents select persuasion tactics (Friestad and Wright, 1994). Persuasion is both complex and impersonal. Persuasive messages engage the audience quickly and are developed for a wide variety of audiences (Mikolajczak-Degrauwe and Bregman, 2013). While the actual delivery of advertisements has changed and shifted towards social media, the principles of effective persuasion have not. The subject of persuasion knowledge through advertising techniques has been an ongoing concern for researchers, especially within the past few decades (Mikolajczak-Degrauwe and Bregman, 2013). In a study assessing persuasion knowledge and attitudes toward advertisements, individuals with higher persuasion knowledge exhibit less positive attitudes towards advertising which can subsequently prevent them from engaging with an advertisement (Mikolajczak-Degrauwe and Bregman, 2013). Moreover, high scores of skepticism and persuasion knowledge towards advertising among consumers concluded a need for advertisers to modify their practices in order to gain more advertisement views from consumers (Mikolajczak-Degrauwe and Bregman, 2013). As stated previously, text variance is just one of the many ways advertisers can modify advertisements to draw attention.

\subsection{Literature Gap}

For those born between 1997 and 2015, Instagram is the second most popular social media application on the market. Facebook, being the most popular, has been previously researched, and it is not relevant to upcoming generations due to the large social media age gap (Anderson, 2021). Moreover, 18-25-year-olds spend an average of three hours a day on social media (Metev, 2020). There have been studies of advertising analysis within other forms of media that were conducted to see the influence of the Persuasion Knowledge Model on online advertisements (Mohapatra, 2017). Various studies have been undertaken in the field of persuasion knowledge primarily dealing with Facebook or other media sources such as newspapers, television, and radios. However, there has yet to be a study done with young adults ages 18-25, let alone an Instagram advertisement analysis. In a critical comparative survey, the research gap discovered that advertisements need future studies to go deeper in understanding how they can attract customers and which platforms are needed to achieve these intended purposes (Abdelaal, 2014). This new topic of interest may potentially initiate new areas of future research. Over $78 \%$ of young adults use Instagram, and of that $78 \%$, about $95 \%$ of teens and young adults have made an online purchase before (Anderson, 2021). Using the Persuasion Knowledge Model, this study aims to look deeply into the language employed in the discourse of advertising and persuasive tactics behind the advertisement. This leads to the research question: To what extent does the Persuasion Knowledge Model and text variance affect Instagram advertisements for 18-25-year-olds living in Pennsylvania? Based on previous research, it was hypothesized that those with lower persuasion knowledge will select the advertisement with the most influential text (ad 3). When an ad has text promoting a sale or a "last chance" opportunity, it is believed that the participants with lower persuasion knowledge will be more inclined to select the ad 3 than the average consumer (Liang, 2018). However, it is noted that those with a higher awareness of persuasion knowledge are less likely to be influenced (Liang, 2018). Although those statistics were for people ages 30 and over, it is possible that since they could have been exposed to more ads than those aged 18-25, their persuasion knowledge is higher. In total, the results exhibited consumers' product knowledge as positively correlated with Instagram advertisements (Liang, 2018). These factors may have altered the previous studies and the discovery of new ideas that can help positively impact Instagram ads in order to increase advertisement awareness within the app.

\section{Methodology}




\subsection{Sampling Method}

The three ads chosen to be researched were selected due to the need for persuasive text based on the Persuasion Knowledge Model. Influential text and text variance were the persuasive tactics surveyed for this study. Influential text is the language used by companies to influence their buyers such as "BOGO" or "limited time only" and text variance is the amount of influential text underneath an advertisement The first ad had the least influential text and promotions to lure the buyer into the online store. The second ad had less text/ promotions than the third, but more than the first. Finally, the last advertisement had the most influential and persuasive text. All advertisements were for both genders and the specified ages (18-25-year-olds) because it allows for a representative advertisement that can be relatable to an increased number of people. In Figure 1, the sponsored ad chosen for the study was from Nordstrom's Department store promoting Nike Blazer Mid ' 77 Infinite Sneaker. This advertisement was sourced from Instagram and appears often on young adults' Instagram feeds (AdEspresso, 2021). The advertisement was chosen from Instagram because the study is solely based on Instagram advertisements only. Before deciding to choose just one ad for the study, a collection of advertisements from Instagram stories were evaluated. Images were eliminated if they included models or people, for those images may entice and be favored instead of the other ads in the study. It was ultimately decided to use only one advertisement and alter the amount of influential text underneath. This method helped to eliminate the bias participants may show towards certain clothes and styles. Figure 1 shows the exact advertisements used and their variations for the study.

FIGURE 1: Instagram advertisement and its text variation used in the survey.

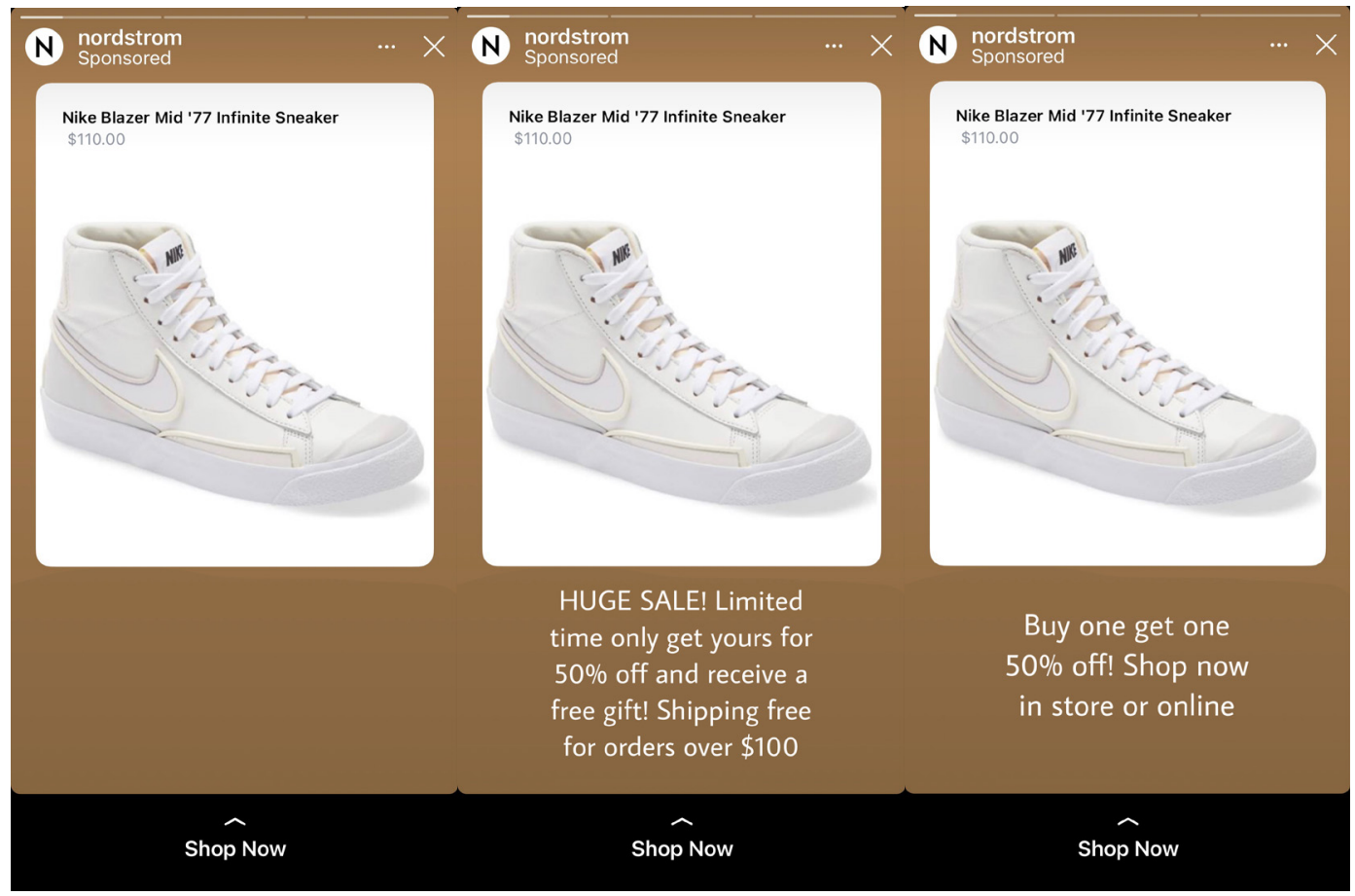

\subsection{Survey Method}

Despite the recommendation from Friestad and Wright to use multiple methods, the dominant method of measuring the Persuasion Knowledge Model was through a survey, with few qualitative approaches (Ham, 2014). The initial scale of the research included personal information and persuasion knowledge-related questions (Hosein and 
Hasanpoor, 2018). No specific individuals were identified in the study, for the only reason they had to identify themselves was to sign the consent form and insert their age for it to be used as a control variable. The questions were derived from peer-reviewed research by Anna Borisova and Ravi Shankar Bhakat. This survey is divided into five parts. In part one, the participants are required to sign and agree to the terms and conditions in addition to the consent forms. Part two consists of fundamental questions in relation to their experiences with online shopping and advertisements on Instagram. This section looks at the respondents' perception, importance, and satisfaction with online shopping. Part three will deal with questions surrounding persuasion knowledge, while part four discusses the internal/ and external factors that contribute to persuasion techniques. Part five includes the three chosen ads and questions that concern them. A Likert 5-point scale was used for the bulk of the survey responses. This scale is a psychometric response scale in which responders specify their level of agreement to a statement typically in five points, ranging from one meaning "strongly disagree, very unimportant, very dissatisfied" to five meaning "strongly agree, very important, very satisfied" (Muruganantham and Bhakat, 2013). This scale was used in multiple peerreviewed articles in this field, thus being the most fitting for what this research aimed to observe (Muruganantham and Bhakat, 2013). The options each participant chose helped to determine where they fell on the persuasion knowledge scale.

To inform individuals residing in Pennsylvania, who were also in the appropriate age range about the study, a sponsored advertisement was put out on Instagram, Twitter, and Facebook. Figure 3 is the exact flyer put up on social media platforms. Though the survey was based on Instagram advertisements, the flyer was placed on other media sites in order to gather more results of the study. This did not create any issues since it was a requirement of the study to be an active user on Instagram. Nevertheless, Instagram was the main platform where survey participants viewed the flyer. To access the survey on Instagram, there was a website link in the bio, a section under an account's username where you can include designated information about yourself and/or your brand. This method generated 174 clicks to the survey page. After being available for two weeks, the survey total was 49 participants. All 49 survey responses from all media sites fit the criteria and were allowed to be included in the study. Below in Table 1 is a report that shows the Instagram users targeted broken down by age and gender, who viewed the ad the most (reach), and the number of times the ad was shown to them (impressions).

FIGURE 3: Advertisement uploaded to social media. 


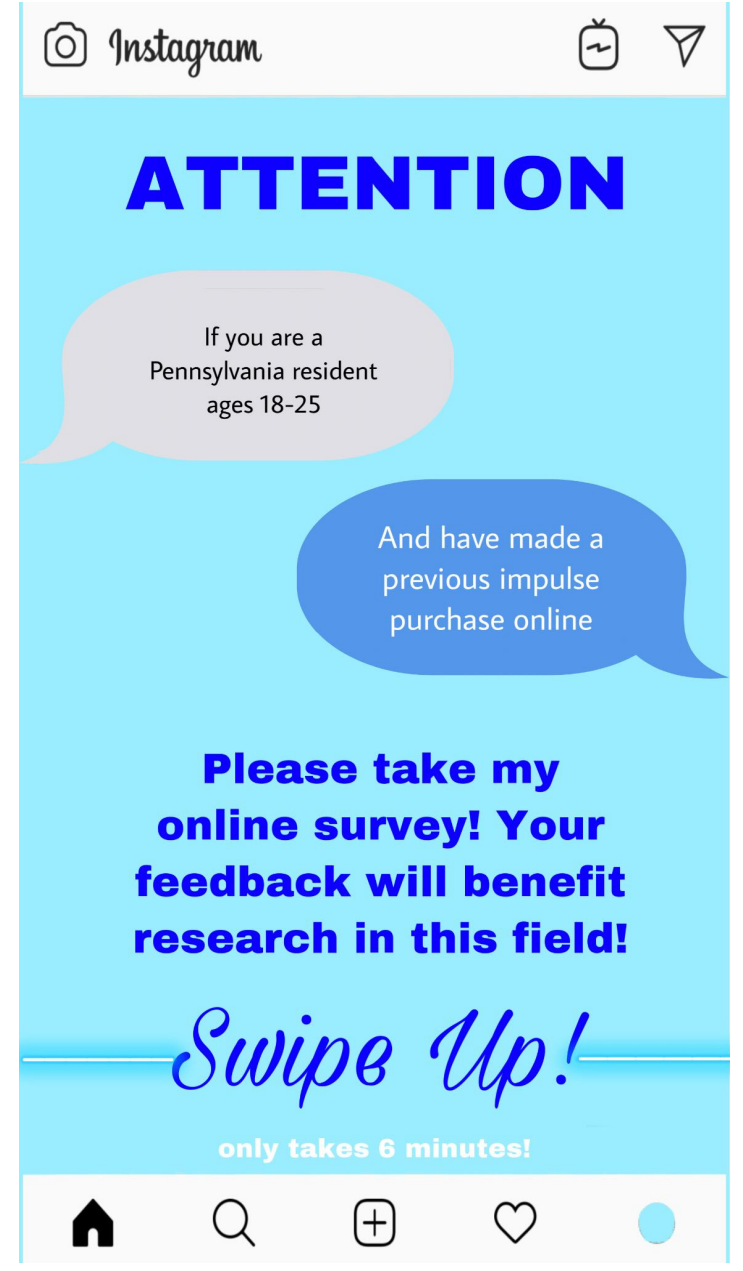

Table 1. Gender Ratio and Instagram Survey Statistics

Instagram Gender Statistics

\begin{tabular}{|l|l|r|r|r|}
\hline Age & Gender & Reach & Impressions & Link Clicks \\
\hline $18-25$ & Female & 4,587 & 6,014 & 50 \\
\hline $18-25$ & Male & 2,637 & 3,130 & 8 \\
\hline $18-25$ & Uncategorized & 70 & 111 & 1 \\
\hline Total & & 7,294 & 9,255 & 59 \\
\hline
\end{tabular}

\subsection{Statistical Analysis}

To perform a proper data and statistical analysis, a data collection process was necessary. This analysis mimics the one utilized in Ravi Shankar Bhakat's study, which measures the correlation between persuasion knowledge and television advertisements. A statistical analysis was conducted within the survey data to see which advertisements appealed to most users and why. Statistical analyses are used while investigating trends, patterns, and relationships using quantitative data. The data in parts two through four from the survey analyzed the participants' knowledge of 
the Persuasion Knowledge Model and the fifth section of the survey determined which advertisement was chosen. Furthermore, the collected information from the survey was analyzed to show the probability of the participant being influenced by advertisements through Instagram. The data was compiled and thoroughly inspected through the use of a mathematical equation, the Pearson product-moment correlation coefficient analysis.

Given that persuasion knowledge covers a multitude of beliefs and behaviors, there is not a specific method of measuring persuasion knowledge to see whether or not it has been activated (Campbell and Kirmani, 2008). Instead, the researcher may choose to decide how the persuasion knowledge is measured (Campbell and Kirmani, 2008). To measure the scale of persuasion knowledge, a categorization of each individual as being high persuasion knowledge, medium persuasion knowledge, and low persuasion knowledge. To be in the high category, the individual must select "always, mostly, somewhat, usually, or very" to more than $50 \%$ of the survey questions. To be in the medium category, the respondent must have chosen "sometimes or neutral" to more than $50 \%$ of the questions. And finally, for the individual to be in the low category, they must have clicked "unlikely, rarely, or never" for more than $50 \%$ of the questions. In essence, this scale was helpful for measuring positive and negative responses to persuasion techniques (Friestad and Wright, 1994). Additionally, this scale remains relevant for both adults and adolescents because it was developed from interviews with adults and successfully applied to youth (Friestad and Wright, 1994). Since the questions were derived from other researchers in the field of persuasion knowledge, the responses allow me to group the individuals into these three groups. The data further analyzed their persuasion knowledge by viewing their responses in the fifth section of the survey, which tested their persuasion knowledge for choosing an advertisement.

\subsection{Content Analysis}

A content analysis was used to determine the presence of certain words, themes, or concepts within some given qualitative data, i.e., text (Bates, 2019). Using content analysis, researchers can quantify and analyze the meanings and relationships of certain words, themes, or concepts (Bates, 2019). Since the verbal data must be converted from the survey into a Pearson product-moment correlation coefficient, there needed to be a way to convert the qualitative data into quantitative data. The content analysis was done prior to conducting the Pearson product-moment correlation coefficient. This quantitative data was formulated in the software IBM SPSS Statistics (SPSS) to produce an outcome. SPSS was used for the research process to perform the complex statistical data analysis because doing it by hand would be inconvenient. By using SPSS, Pearson product-moment correlation coefficient analysis, and the Persuasion Knowledge Model, it was anticipated for a correlation to clearly show the amount of Instagram users who potentially display persuasion knowledge towards advertisements on Instagram. The survey was converted from qualitative data to quantitative data by taking both the variables and inputting them into SPSS. In column one, the level of persuasion knowledge was input for each participant, and in column two was the advertisement each person chose. While being put into the Pearson correlation coefficient, the data was processed through the Pearson equation to transform the data from qualitative to quantitative.

\subsection{Pearson Product-Moment Correlation Coefficient Analysis}

In addition to the other methods of analysis, this study ran a Pearson product-moment correlation coefficient analysis. As used in Ying-Ping Liang's study, this test measures the strength of a linear association between two variables and is denoted by $r$ (Lund Research 2018). Variable $r$ measures the strength of the association between the two variables, or how closely related the two variables actually are (Lund Research, 2018). The coefficient $r$ indicates how far away these data points are from this line of best fit (Lund Research, 2018). A Pearson product-moment correlation attempts to draw a line of best fit through the data of two variables; in this case, the variables are the participants' persuasion knowledge and the advertisement the Instagram advertisements they chose. However, it is only viable to use Pearson's correlation if the data "passes" four out of the seven assumptions required for Pearson's correlation to give a valid result, which this study did (Lund Research, 2018). This test was used to measure the correlation between viewing the 
advertisement, filling out a persuasion knowledge survey, and the likelihood of choosing an advertisement depending on where the participant falls on the scale of persuasion knowledge. There are three different types of correlations that may arise from the data. A positive correlation is shown when all the points and lines of best fit from the data are positive and going upward. When the opposite occurs and the plotted points are negative, there is a negative correlation. If the plotted points are scattered and not showing any order or connection, there is no correlation. See figure 2 for visual aid.

FIGURE 2: The three types of correlations that may result from the Pearson correlation. Lund Research 2018

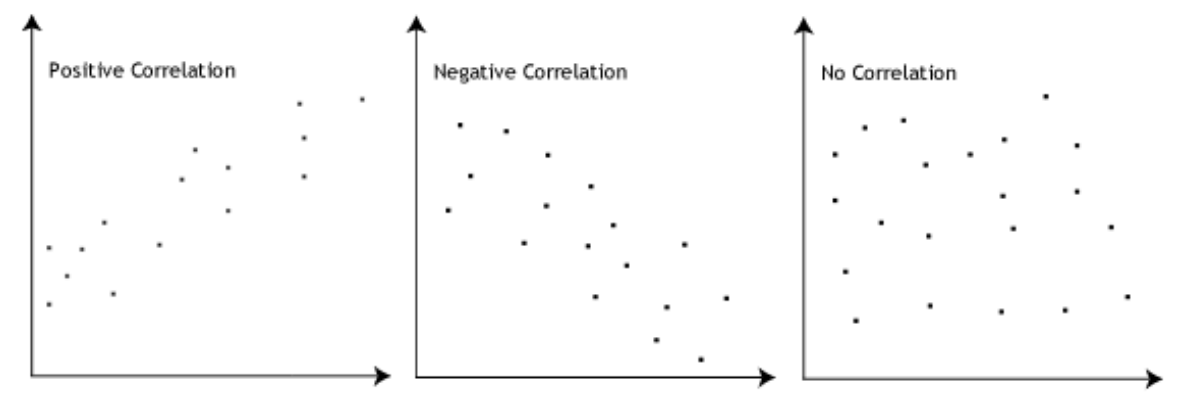

\section{Results}

\subsection{Statistical Analysis}

After gathering my data from the survey, the data was input into a statistical analysis to find the relationship between the level of persuasion knowledge each participant had and the advertisement chosen in the survey. The presented data refuted my initial hypothesis. Figure 3 shows that those with low persuasion knowledge choosing advertisement three, those with high persuasion knowledge were more likely to select it. In turn, those with low persuasion knowledge were more likely to select advertisement one, which goes against the foundation of the Persuasion Knowledge Model. Finally, advertisement two was chosen the most by those with medium persuasion knowledge.

FIGURE 3: Pie chart showing percentages of each advertisement chosen 


\section{Advertisement Statistical Analysis}

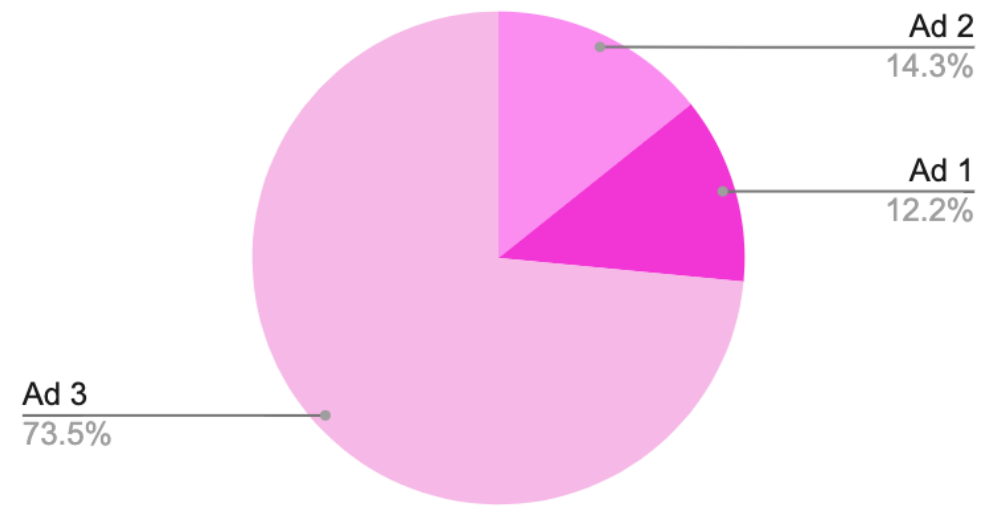

\subsection{Pearson Product-Moment Correlation Coefficient Analysis Results}

To run a Pearson Correlation Coefficient test, there must be at least two variables that are measured in order to reach an appropriate correlation. The two independent variables in this study were each participants' persuasion knowledge measurement and the advertisement they chose in the survey. It was hypothesized that there would be a positive correlation between the two variables, which meant that the lower a participant's persuasion knowledge, the more likely they will select an ad with a greater amount of text. In contrast, however, the results showed there was a slight negative correlation as shown in Table 1. The measurement of the strength of a linear association between the two variables is $r=-0.243$. Any variable between -0.1 and -0.3 is considered a small negative correlation. This means that as one variable increases in value, the second variable decreases in value (Statistics Help For Students, 2008).

Variable $N$ simply shows how many survey participants were surveyed. Sig (2-tailed) is the p-value evaluating no statistical significance that exists against an alternative that the mean is not equal to 50. (UCLA 2021). If the p-value is less than the pre-specified alpha level, usually .05 or .01, it is generally concluded that the mean is significantly different from zero (UCLA, 2021). A p-value is a number between 0 and 1, representing the probability that this data would have arisen if the null hypothesis was supported (Fenton and Neil, 2012). As concluded, the pvalue discovered by the study was $\mathrm{p}=0.131$. However, since the $\mathrm{p}$-value is higher than .05 , this suggests the correlation is not statistically significant. A p-value greater than 0.05 also indicates strong evidence for the occurrence of a null hypothesis. The null hypothesis states that there is no relationship between an independent and dependent variable. Nevertheless, this outcome could be due to an experimental or sampling error (Lund Research, 2018). Furthermore, increases or decreases in one variable did not significantly relate to increases or decreases in the second variable (Statistics Help For Students 2008). Overall, this study did find a correlation, however, the correlation was not statistically significant. (Lund Research, 2018).

Table 2. Correlation Between Persuasion Knowledge and Advertisements 


\section{Correlations}

\begin{tabular}{|l|l|r|r|}
\hline & & Persuasion Knowledge & Advertisements \\
\hline Persuasion Knowledge & Pearson Correlation & 1 & -0.219 \\
\hline & Sig. (2-tailed) & 49 & 0.131 \\
\hline & N & 49 \\
\hline Advertisements & Pearson Correlation & -0.219 & 49 \\
\hline & Sig. (2-tailed) & 0.131 & 49 \\
\hline
\end{tabular}

\section{Conclusion and Implications}

After conducting the survey and analyzing the results, this study was able to provide the field of persuasion knowledge and social media advertisements with future implications. Previous research implies that the higher the participant's persuasion knowledge, the greater probability that they will choose an advertisement with less influential text (Liang, 2018). Findings from this study add an alternative view on this issue, for the results concluded that there was a slight negative correlation. This also refutes the outcome of others in the field of persuasion knowledge because the model depicts a positive correlation between the two variables. These conclusions open a new line of inquiry important to this field because there has yet to be a study directed through Instagram advertising and young adults. The data acquired implies that there is some sort of correlation between persuasion tactics regarding text underneath advertisements.

With these findings, companies have a better understanding of how persuasion knowledge techniques affect how users view their advertisements and the power of text variance. They are able to take away information from this study and apply it to their advertisements in order to reach certain target audiences. In addition, Instagram users are made aware of the techniques advertisers use to draw customers into their online stores. They are made more aware of techniques marketers use, which can help them increase their persuasion knowledge.

\section{Limitations}

This study was not exempt from limitations. The age of participants was a restriction because those participating must have been within the ages of 18-25 to be considered as a part of the study. Working with minors under 18 is more difficult due to consent issues. However, it is still plausible that some participants lied about age or survey responses just to be included within the study. Additionally, results from this study may not apply to people other than the specified ages. Similar to age restriction, there is also the limitation of area. The survey was conducted in Pennsylvania, so data may differ from other states or regions. This study was unable to gather enough data, so the conclusions can not be generalized for all areas not surveyed. In order to gain data from all areas in Pennsylvania, the survey would have needed a few responses from each county in order to gain a broader perspective. Unfortunately with limited data responses, the study was not able to reach all counties of Pennsylvania. These new findings may be drastic and statistically significant depending on the area they are conducted. Since some areas in the United States have more affluence and a different social structure, the results may help advertisers in those areas appeal to both the wealthy and middle class.

Another limitation regarding the advertisements is the text/variance put under each one as well as where they are placed (first, second, third). The placement of the ad could have altered the study's findings because some participants are not inclined to read all three ads and therefore didn't read the last two ads. There were only 49 surveys gathered during the data collection process. With that being said, the sample size was not large enough in order to generalize this study's conclusions. 


\section{Further Research}

The findings of the analysis have not only presented me with a conclusion, but also a gap in the field. Further research should touch on other aspects of social media. Since Instagram advertisements were analyzed, future studies should look at other methods of advertising whether it be on Twitter, Facebook, Pinterest, or other social media networks because of their different target demographics. Future research should target other upcoming apps with advertisements directed at your age range, such as TikTok and Snapchat.

In addition, newer research should use different advertisements and determine if the different ads change the outcome of the survey. This can test to see whether the advertisement changes the persuasion knowledge portrayed while viewing the advertisement or if it makes no difference. In another variation of this study, researchers can change the order the viewer sees the advertisements. For instance, the ad with the most text can be placed first and see if it makes a difference in conclusions because it was predicted that some participants would choose advertisement 1 because there was less to read.

New studies should also expand on a Pearson Correlation Coefficient Test or other statistical analyses with more variables rather than just two. Using more than two variables will allow researchers to answer more research questions with minimal additional effort. More specifically, variables are important because slight variations in the experimental setup could strongly affect the outcome being measured.

Further research should focus on specifically males or females and what differences arise when looking at each gender's response to persuasion techniques within Instagram advertising. This discrepancy can help advertisers who are broadcasting advertisements to a certain area to increase their advertisement views in social media. A male may potentially have different persuasion knowledge than a woman, so the approach for how the ads come across social media for both genders may require different specifications. Another area for potential research may lie in different ads or more than one ad should be studied to discuss the differences to the current findings to help advertisers see which methods or ads consumers view as more attractive. Research could also expand to account for a bigger area or state. Vice versa, new data would be beneficial to study specific local businesses. This research would help smaller growing businesses take advantage of advertising on Instagram by boosting views depending on the audience's persuasion knowledge. With that in mind, future researchers could apply it to one brand and see how the brand benefits or does not benefit from the advertisement.

Also, other age gaps, like those in their mid-twenties or younger, may lead to differences from the data that was found, so researchers should expand on the topic's age range. Future research should expand on children and teenagers and how those findings differ from those of young adults. In addition, what 18-25-year-olds may see and interact with on Instagram, may not be the same as the next generation of young adults in the same age range. Further research should conduct a study that analyzes different generations of Instagram users to see which range is most persuaded by different advertisements. Along with this, future generations should be tested if their persuasion knowledge increases or decreases with changing culture.

\section{Closing Remarks}

In total, this research study aimed to find the correlation between Instagram user's persuasion knowledge and advertisements. It was initially hypothesized that this study's findings would reflect those of Ying Ping Liang's study and support the Persuasion Knowledge Model. Conversely, the findings of this study concluded that there was a slightly negative correlation. This correlation indicated that the users with the lowest persuasion knowledge were most likely to select the advertisement with the least text (advertisement 3). Moreover, this finding refuted my hypothesis which predicted that users with the lowest persuasion knowledge will be more likely to select the advertisement with the most text according to the Persuasion Knowledge Model. Due to the p-value drawn from the Pearson correlation 
test, the correlation between the two variables was not statistically significant. This indicated that a null hypothesis had arisen. With these new understandings, companies and Instagram users can be more aware of the persuasion techniques employed within the advertisements on Instagram.

\section{Acknowledgments}

First and foremost, I would like to do a special thank you to my teacher and advisor, Mr. Robert Hodgson. I am forever thankful for your guidance, faith, and patience during the entire process. Additionally, I would like to extend my gratitude to Mrs. Sandy Means and Mrs. Katie Maccaglia for the constant support and positivity surrounding my research. Last but certainly not least, I want to thank The Education Center of Millcreek Township School District for aiding my project financially.

\section{References}

Abdelaal, N. (2014, November 30). Advertisement analysis: A comparative critical study. Retrieved February 22, 2021, from https://eric.ed.gov/?id=EJ1128511

AdEspresso. (2021, January 5). 53 Amazing Instagram Ads Examples To Inspire You. AdEspresso.https://adespress o.com/blog/37-instagram-ads-examples/.

Anderson, M., \& Jiang, J. (2021, April 7). Teens, Social Media \& Technology 2018. Pew Research Center: Internet, Science \& Tech. https:/www.pewresearch.org/internet/2018/05/31/teens-social-media-technology-2018/.

Bhandari, P. (2021, April 19). Control Variables: What Are They and Why Do They Matter? Scribbr. https://www.sc ribbr.com/methodology/control-variable/.

Campbell, M., \& Kirmani, A. (2008). I know what you're doing and why you're doing it: The use of Persuasion Knowledge Model in consumer research. Research Gate. https://www.researchgate.net/publication/269 691369_I_know_what_you're_doing_and_why_you're_doing_it_The_use_of_Persuasion_Knowledge_M odel_in_consumer_research.

Charlton, G. (2021, February 19). What is Cart Abandonment? SaleCycle. https://www.salec ycle.com/blog/feature d-en-us/what-is-cart-abandonment/.

Columbia University Public Health. (2019). Content Analysis. Public Health Methods. https://w ww.publ ichealth.columbia.edu/research/population-health-methods/content-an alysis\#: :text=Content $\% 20$ ana lysis $\% 20$ is $\% 20 a \% 20$ research,qualitative $\% 20$ data $\% 20$ (i.e. $\% 20$ text).\&text $=$ Researchers $\% 20$ can $\% 20$ then $\% 20 \mathrm{make} \%$ 20inferences,time $\% 20$ of $\% 20$ surrounding $\% 20$ the $\% 20$ text

Direct Packaging. (2020, November 28). What is E-Commerce? [E-Commerce Guide Series]. Direct Packaging Solutions. https://www.dpack.co.uk/blog/what-is-e-commerce\#: :text=E \%2DCommerce\%20\%2D\%20n oun $\% 20 \% 2 \mathrm{D} \% 20$ commercial,online $\% 20$ or\%20over\%20 the $\% 20$ internet.

Friestad, M., \& Wright, P. (1994). The Persuasion Knowledge Model: How People Cope with Persuasion Attempts. Journal of Consumer Research, 21(1), 1-31. Retrieved May 14, 2021, from http://www.jstor.org/stable/24 89738 
Grand View Research. (2020, May). E-commerce Market Share, Growth \& Trends Report, 2020-2027. Grand View Research. https://www.grandviewresearch.com/industry-analys is/e-commerce-market.

Ham, Chang-Dae \& Nelson, Michelle \& Das, S.. (2015). How to measure persuasion knowledge. International Journal of Advertising. 34. 17-53. 10.1080/02650487.2014.994730.

Hasanpoor, H., Tojari, F., \& Nikaeen, Z. (2018). Validation of Expanded Scale of Impulse Buying in Sports, 1-10. http://www.aassjournal.com.

Holmstrom, K. (2020). The Importance of Typography in Advertising. Diggles Creative. https://www.diggle screative.com/blog/importance-of-typography-in-adv ertising.html.

Hsieh, H.-F., \& Shannon, S. E. (2005). Three Approaches to Qualitative Content Analysis. Qualitative Health Research, 15(9), 1277-1288. https://doi.org/10.1177/104973230 5276687

Kus, P. (2020, December 09). Instagrowth: The history of advertising on Instagram. Retrieved December 12, 2020, from https://www.marketingsupply.co/blog/instagrowth-history-of-advertising-on-Instagram/

Leong, L.-Y., Jaafar, N.I. and Sulaiman, A. (2017), "Understanding impulse purchase in Facebook commerce: Does Big Five matter?", Internet Research, Vol. 27 No. 4, pp. 786-818. https://doi. org/10.1108/IntR-04-2016-0107

Liang, Ying-Ping. (2012). The Relationship between Consumer Product Involvement, Product Knowledge, and Impulsive Buying Behavior. Procedia - Social and Behavioral Sciences. 57. 325-330. 10.1016/j.sbspro.20 12.09.1193.

Liu, W., Lin, C., Lee, Y., \& Deng, D. (2012, September 04). On gender differences in consumer behavior for online financial transactions of cosmetics. Retrieved February 2, 2021, from https://www.sciencedirect.com/scie nce/article/pii/S0895717712002336

Lund Research. (2018). Pearson's Product-Moment Correlation using SPSS Statistics. Pearson's Product-Moment Correlation in SPSS Statistics - Procedure, assumptions, and output using a relevant example. https://statist ics.laerd.com/spss-tutorials/pearsons-product-moment -correlation-using-spss-statistics.php.

Maryville University. (2021, March 3). The Evolution of Social Media: How Did It Begin and Where Could It Go Next? Maryville Online. https://online.maryville.edu/blog/evolution-social-media/.

Metev, D. (2021, May 7). How Much Time Do People Spend on Social Media in 2020? Review42. https://review 42.com/resources/how-much-time-do-people-spend-on-social-media/.

Mikołajczak-Degrauwe, K., \& Brengman, M. (2014). The influence of advertising on compulsive buying - The role of persuasion knowledge. Journal of behavioral addictions, 3(1), 65-73. https://doi.org/10.1556/JBA.2.201 3.018

Mohapatra, B. (2017). Internet users' attitudes towards online targeted advertisements. Journal ism and Mass Communication, 5(12), 1-70. https://doi.org/10.17265/2160-6579/2 015.12.001 
Neil, M., \& Fenton, N. (2012). Chapter 1 of "Risk Assessment and Decision Analysis with Bayesian Networks." Correlation coefficient and p-values: what they are and why you need to be very wary of them. http://www.ee cs.qmul.ac.uk/ norman/blog_articles/p_valu es.pdf.

Null Hypothesis - Overview, How It Works, Example. Corporate Finance Institute. (2020, November 23). https://corp oratefinanceinstitute.com/resources/knowledge/other/null-hypothesis-2/.

Omnicore. (2018, November 8). Content Marketing Services by Omnicore ${ }^{\mathrm{TM}}$. Omnicore. https://www.omnicoreagen cy.com/services/content-marketing-services/.

Question Pro. (2020, May 4). Pearson correlation coefficient: Introduction, formula, calculation, and examples. QuestionPro. https://www.questionpro.com/blog/pearson-correlation-coefficient/.

Saleh, K. (2018, April 11). The State of Impulse Buying Persona - Statistics and Trends. Invesp. https://www.inves pcro.com/blog/impulse-buying/.

Statistics Help For Students. (2008). How do I interpret data in SPSS for Pearson's $r$ and scatterplots? http://statis tics-help-for-students.com/How_do_I_interpret_data_in_SPSS_f or_Pearsons_r_and_scatterplots.htm\#.YKF q_WZucr1.

UCLA Statistical Consulting Group. (2021). SPSS Annotated Output T-test. IDRE Stats. Http s://stats.idre.ucl a.edu/spss/output/t-test/\#: :text=Sig\%20(2\%2Dtailed)\%E2\%80\%93,05\%20or\%20.

What is E-Commerce? [E-Commerce Guide Series]. (2020, November 28). Retrieved April 1, 2021, from https://www.dpack.co.uk/blog/what-is-e-commerce\#: :text=E\%2DCommerce\%20\%2D\%20no un $\% 20 \% 2 \mathrm{D} \% 20$ commercial,online $\% 20$ or $\% 20$ over\%20the $\% 20$ internet.

Wu, Y. (2009). "Seeing is believing?": A persuasion knowledge model on attitudinal evaluations in advertisements (Master's thesis, Lingnan University, Hong Kong). Retrieved from http://dx.doi.org/ 10.14793/mkt_etd.2 